Planetary Systems in the Universe - Observation, Formation and Evolution

Proceedings IAU Symposium No. 202, (C)2004 IAU

Alan Penny, Pawel Artymowicz, Anne-Marie Lagrange, \& Sara Russell, eds.

\title{
Detecting Earth-Uranus Class Planets with the Space Mission COROT
}

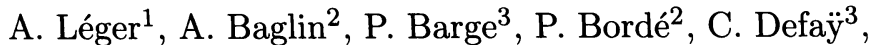 \\ M. Deleuil ${ }^{3}$, D. Rouan ${ }^{2}$, J. Schneider ${ }^{2}$ and A. Vuillemin ${ }^{3}$ \\ ${ }^{1}$ Institut d'Astronomie Spatiale - CNRS, Orsay, France \\ ${ }^{2}$ DESPA \& DARC, Observatoire de Paris-Meudon, Meudon, France \\ ${ }^{3}$ Laboratoire d'Astrophysique de Marseille - CNRS, Marseille, France
}

\begin{abstract}
The space mission COROT can be considered as a DARWIN/TPF precursor, since it will contribute to the pre-DARWIN/TPF effort with its dedicated exoplanet program. COROT will survey more than 50 thousands stars, each one during five months, in order to detect transits of planets. Despite of the modest size of the telescope, the photon-noise limited performances may lead to the detection of several tens of "hot jupiters" and, more important, of a few planets of the Earth-Uranus class, i.e. with a size of typically 1.5 to 4 Earth radius. In the basic mode of operation (characterization of a planet by at least 3 detected transits), only planets with a short orbital period, and thus rather "hot" ones $(\mathrm{T}=600 \mathrm{~K})$, should be discovered. However, one of the COROT's features is its capability to provide a wavelength dependent information, thanks to a prism in the optical path.
\end{abstract}

\section{Introduction}

A sensible approach of the search and study of extra-solar planets around main sequence stars is: a) searching for giant exoplanets, $b$ ) searching for terrestrial ones, c) performing their spectroscopic analysis, with emphasis on Earth-type ones. The first step has been made with the discovery of 51 Peg b in 1995 (Mayor, Queloz, 1995). In fact, it has been preceded by a photometric observation by Hipparcos of a planetary transit in front of the star HD 209458, several years before, but recognized as such only recently (Robichon \& Arenou, 1999). Since this first discovery, there has been a dramatic increase of the number of planets of Jupiter's class detected by means of radial velocity (RV) technique. However, presently we miss unbiased statistics of these planets. Step (b) is accessible to the COROT mission only (or to similar ones, as the ESA mission Eddington (2007-09), or the NASA mission Képler but which is not approved). The step (c) is the main goal of DARWIN, possibly the most ambitious mission ever studied by ESA or its sister NASA mission, TPF.

Concerning giant exo-planets, the ESA 2012 Cornerstone, GAIA, should detect about 50000 giant planets due to the huge number of stars which are studied by astrometry, at the 10 micro-arcsec level. It should provide an excellent statistics on giant planets but cannot detect Earth-like ones. COROT, in its exoplanetary program (Rouan et al., 1999), will search for planetary transits in front of stars by performing stellar photometry with an accuracy which is not accessible from the ground. Presently, we do not know the statistical 
distribution of earth-like exoplanets as a function of their size. COROT could demonstrate the "existence theorem". Now, this piece of information is crucial for DARWIN/TPF whose main goal is to perform the spectroscopy of these objets.

COROT will not provide a target list for DARWIN/TPF because it will only detect earth-type planets with orbit almost edge-on. It must monitor a large number of stars in a given field of view (few square degrees) and therefore distant ones, typically at $500 \mathrm{pc}$. DARWIN/TPF will concentrate on the nearest stars and spend the beginning of its mission searching for those that have planets. If COROT have provided the statistical information on the fraction of stars that have planets of a given size, it will be a most valuable piece of information for optimizing the instrument performance and the searching procedure.

\section{The Exoplanet program in COROT}

The transit method (e.g. Schneider, 1996) has the unique advantage to potentially detect Earth-type planets around solar-type stars, since the amplitude of the variation - typically of $\left(\mathrm{R}_{\oplus} / \mathrm{R}_{\odot}\right)^{2} \approx 10^{-4}$ - is within reach of a photometric experiment in space, monitoring thousands of stars continuously.

\subsection{The mission and instrumental set-up}

COROT (Baglin, 1998) was selected within the frame of the Small Mission Program of the French Space Agency CNES with contributions of other Space Agencies . It will cost typically 60 MEuros. Partners are : CNES, LAS (Marseille), DESPA (Meudon), IAS (Orsay), Austria, ESTEC, ESA, Italy and Belgium. More modest than the ESA's Eddington mission and the US proposed Képler mission (Koch et al., 1996), COROT should be launched first.

The main characteristic of COROT is to perform high accuracy photometry on a wide field. This will permit to fulfill the requirements of the two main scientific objectives of the mission:

a) measuring stellar pulsations on a small set of stars,

b) searching for exoplanets around thousands of target stars.

The two programs share a same instrument, featuring a $27 \mathrm{~cm}$ telescope without obscuration ( 2 off-axis parabola plus a dioptric objective) and 4 cooled ( -40 Celsius) CCD $2048 \times 2048$ from EEV, with two devices per program. The field of view of the 2 exoplanets CCDs is $3.5\left(^{\circ}\right)^{2}$. The platform Proteus provides a pointing stability of 0.15 arcsec. The orbit of COROT is pseudo-polar, quasiinertial at an altitude of $850 \mathrm{~km}$. During the 2.5 years mission, five fields will be observed continuously, each during 150 days. Launch, using a Russian Rockot or a rocket from India, is planned for 2004.

The goal of the Exoplanets program, will be achieved by monitoring continuously 6000 to 12000 stars in each field, with $\mathrm{m}_{V}=11$ to 16.5 , at rather low galactic latitudes $\left(\mathrm{b}_{I I}=5-15^{\circ}\right)$. Each exposure will be 32 sec. In order to cope with the data transmission rate of 900 Mbits per day, data will be coadded on-board during periods of 15 minutes, before being downloaded. Time sampling will be improved, down to 1 minute, for few stars for which a high $\mathrm{S} / \mathrm{N}$ event due to a giant planet will have been previously detected, in order to search for satellites thanks to a possible reflex motion of the planet (Sartoretti 
\& Schneider, 1998). Scattered light from the Earth limb will be maintained at a low level thanks to an afocal telescope design and a long baffle. Zodiacal light level is important but is stable and will dominate the background noise. The expected performances are such that a big earth $\left(\mathrm{R}_{p l} / \mathrm{R}_{\oplus}=1.6\right)$ should be detected against a $\mathrm{m}_{V}=14$ star, with a $\mathrm{S} / \mathrm{N}=4$, on transit events of 10 hours duration. In the basic mode (three transits detected), only planets with an orbital period shorter than 50 days will be unambiguously detected, i.e. "hot" planets $(\mathrm{T}>$ $600 \mathrm{~K})$. The chromatic mode was proposed to partly overcome this limit.

\subsection{The multi-color information}

A transit event should appear as a characteristic downward slot in the light curve, and usually be distinguished from a presumably more chaotic stellar fluctuation. However, some stellar fluctuations could mimic a transit. A color information will help strongly to remove the ambiguity (Rosenblatt, 1971). Transits are essentially achromatic (geometrical effect), while stellar fluctuations are highly chromatic (temperature variations on the stellar surface) as shown by the VIRGO experiment on-board SOHO $\left([\Delta F / F]_{400 \mathrm{~nm}}=2.4 \times[\Delta F / F]_{800 \mathrm{~nm}}\right)$.

A second important advantage of a chromatic information is that ambiguity can be removed in the case of a binary stellar system where a Jupiter-type transit around the faint component can mimic a Earth-type event around the brightest star. The confusion between the two types of events will be the most critical when the two stars have typically 5 magnitudes difference in brightness. In that case their temperatures and colors will be very different and the relative variations in the blue and in the red should give the proper answer. This is a real problem if one considers that $70 \%$ of the stars belong to a binary system.

In order to obtain this chromatic information, a small bi-prism is put a few centimeters above the exoplanet CCDs and produces an on-axis mini-spectrum for each star. Fig.1 illustrates how the PSF obtained by introducing a pair of prisms, head to toe, in the beam can provide this multi-color photometry. Astigmatism generated by the prisms is used to spread the image perpendicular to the dispersion while keeping a sufficient spectral resolution. Summing pixels on defined ranges of this spectrum gives red, green and blue fluxes.

A proper combination of the different color light curves will be used as a discrimination function. We have shown (Defaÿ, 1999; Bordé et al., 2000) that a linear combination of three colors is a powerful discrimination function, when the weights are properly chosen. Fig.2 shows the confidence level of the identification of a transit event, using this chromatic criterium, according to the $\mathrm{S} / \mathrm{N}$ ratio on the integrated (white) flux. As one expects, the higher the $\mathrm{S} / \mathrm{N}$, the higher the confidence level for the identification.

An important consequence of this discrimination by the colors is that dual events, or even unique ones, could be identified as transit, relaxing the condition on a short orbital period of the planet and thus on its distance to the star. This implies, in particular, that COROT can detect Uranus-size planets in the Habitable Zone.

\subsection{Sources of noise, performances}

Numerical simulations of the optical setup and platform pointing, as well as laboratory tests on CCD allowed us to identify the perturbations and the limi- 


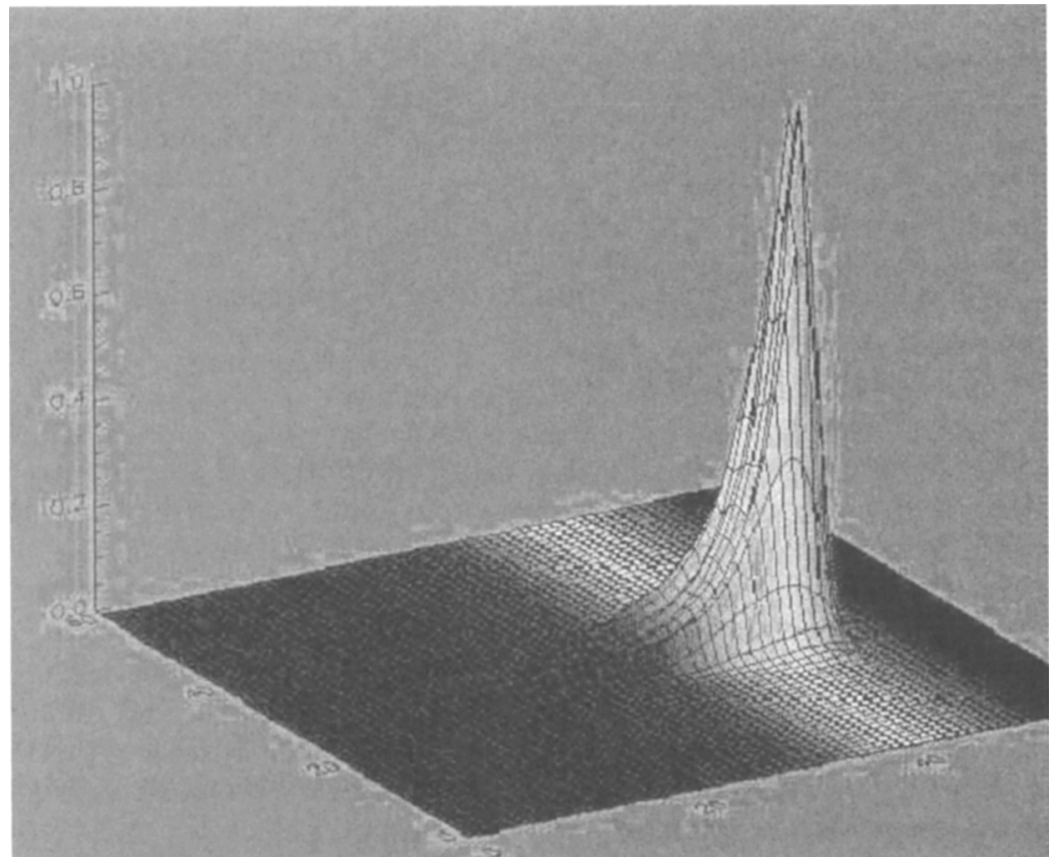

Figure 1. The PSF on the exoplanets channel of COROT. A pair of prisms, mounted head to toe, provides a dispersion in one direction of the CCD at a low spectral resolution, and also contribute, through astigmatism, to spread the light in the perpendicular direction for a better photometry.

tations in photometric performances. They are shown in Fig.3 and as follows: - Pointing errors coupled to non-uniformity of the CCD response (flat field) should not be a limit thanks to the excellent pointing performances of the Proteus platform ( 0.15 arcsec rms).

- Defocusing at an orbital period, due to small distortions of the telescope structure when thermal fluctuations are generated by sun eclipses along the orbit. This may become a noise larger than the photon noise and will require ground post-processing. To reduce this orbital effect, the integration time on one orbit is adjusted to be as close as possible to the actual orbital period.

- Scattered light from the Earth limb: a level of $0.1 \mathrm{e}^{-} \mathrm{s}^{-1}$ pixel $^{-1}$ is expected from simulation with the afocal telescope design and an efficient baffle; even if modulated, this will give a small contribution to the signal and to the noise.

- Zodiacal light: using data from James et al. (1997), we evaluate a maximum contribution to the signal of $10 \mathrm{e}^{-\mathrm{s}^{-1}}$ pixel $^{-1}$; this level is important since its statistical noise will be slightly larger than the read-out noise, but it is stable.

- Nearby variable stars: if a faint variable star is within the photometric diaphragm of one of the target star, its fluctuation may mimic a transit. We have modeled this effect using actual stellar field and found that it should happen in less than $5 \%$ of cases, assuming $30 \%$ of variable stars with $5 \%$ relative variation, a very pessimistic case compared to Hipparcos results (Grenon, 1999). 


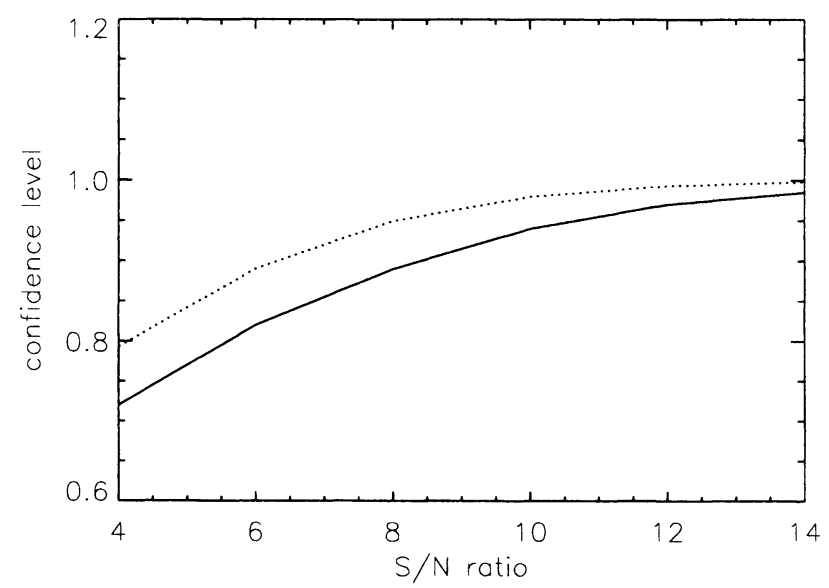

Figure 2. Plot of the confidence level of the discrimination - thanks to the chromatic information - between actual transits and other photometric events versus total flux S/N. Solid line: a single transit with respect to a stellar activity fluctuation; dotted line: a jupiter's transit on a M5 star with respect to an earth's transit on a K5 star (case of binary stars) .

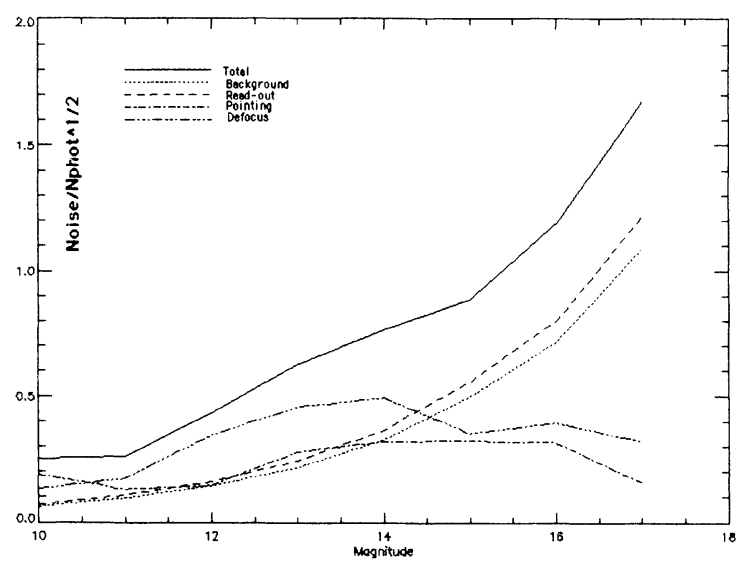

Figure 3. Uncertainties introduced by the different sources of noise, with respect to the photon noise, as a function of the stellar magnitude.

\section{Expectation on the number of planets COROT could detect}

Using models of stellar populations in the Galaxy ( Bienaymé, Robin \& Creze, 1987), and the performances expected with COROT, we have estimated the number of events that should be detected during a two and half year mission. Two cases were contemplated : multi-transits $(\mathrm{N} \geq 3)$ and transits identified by their characteristic colors. For a given couple of stellar apparent magnitude and orbital distance of the planet, one can determine the minimum $\mathrm{S} / \mathrm{N}$ ratio (and 


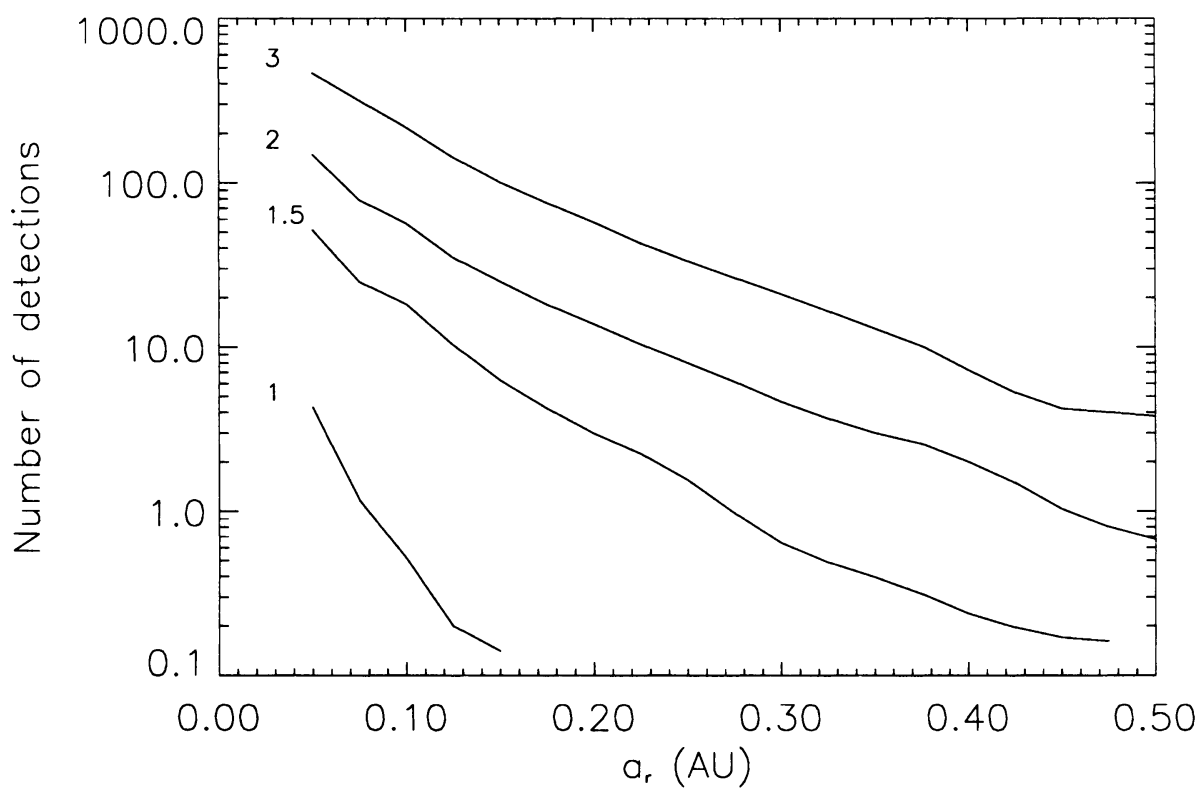

Figure 4. Plot of expected number of planets detected by COROT according to their radii and reduced orbital distances, $a_{r}=a\left(L_{\star} / L_{\odot}\right)^{-1 / 2}$, to the planet. The label on each curve corresponds to the reduced planet radius $\left(R_{p l} / R_{\oplus}\right)$. The expected detections are based on the multi-transit mode (N $\geq 3$ ) only. They are for the $2.5 \mathrm{yr}$ mission, assuming that each star has a $20 \%$ probability to having a planet with characteristics $\left(R_{p l}, a_{r}\right)$. If stars have several planets, the corresponding detection numbers add.

thus size of the planet) allowing a detection. Taking into account the geometrical and temporal probabilities to have - for this orbit - a given number of transits during the observing period, as well as the density of stars of this magnitude and spectral type in the line of sight, the number of expected detections is derived. Since there is no model of the orbits distribution in an "average" planetary system, a presentation of the results is chosen that allows a scaling of the expected detections as a function of different hypotheses on the abundance of planets. the number of detection is plotted as function of the planet radius, $R_{p l}$ and reduced orbital distance, $a_{r}$, assuming circular orbits. Fig.4 summarizes the result of the prediction model in the case where the fields are at $b_{I I}=10$ deg. A false detection probability of $10^{-5}$, per star during the whole mission, is accepted.

Jupiter-type planets. Assuming $\mathrm{R}_{p l}=\mathrm{R}_{J u p}$, we derive that COROT should detect a large sample of hot jupiters popularized by RV measurements. If we take as a basis $\mathrm{T}_{p l}=1300 \mathrm{~K}$ and assume $2 \%$ of stars with a hot jupiter, a fraction derived from RV data, then all of those planets with orbit properly aligned should be detected, i.e. about one hundred for the whole mission. Note that the chromatic information will also bring in a fair statistics on big planets 
in the 0.7 - 1.6 AU range (warm Jupiters). For instance, if Jupiters with $a_{r}$ close to $1 \mathrm{AU}$ are not rare, then a fair number of them should also be detected $(\approx 30$ if they are abundant at the level of $20 \%$ per star).

Earth-Uranus class planets. The fraction of stars with Earth-like planets is very uncertain. However, if we assume that large rocky planets, i.e. with $1.5<$ $\mathrm{R}_{p l} / \mathrm{R}_{\oplus}<2.2$ (Wuchterl, Guillot and Lissauer, 1999), are rather frequent (20\% probability), then, with the same galactic model and in the basic detection mode of COROT (multi-transits), up to 50 planets could be detected if their (reduced) orbital distance is between .05 to $.20 \mathrm{AU}$.

With the chromatic information, mono or bi-transits with sufficient $\mathrm{S} / \mathrm{N}$, typically 12 , can also be identified. The number of detected planets could reach a ten for Uranus-size planets in the 0.5 - 1 AU range of orbital distance, i.e. possibly in the Habitable Zone (the model by Chyba, Whitmire and Reynolds, 1999 , is assumed).

All these information would be very valuable for designing the DARWIN and TPF missions.

\section{References}

Baglin, A. 1998, in "New Eyes to see Inside the Sun and Stars", Deubner et al. (eds), p. 301

Bienaymé, O., Robin, A., \& Crézé M. 1987, A\&A, 180, 94

Bordé, P., Rouan, D., \& Léger A. 2000, submitted

Chyba, Whitmire, \& Reynolds 1999, in "Protostars and Planets IV" (in press)

Defaÿ, C. 1999, in "Documentation for the preparation of the COROT space mission" (LAS - COR.SCI 990004.01), in French.

Grenon, M., 1999, in Highlights of Astronomy, Vol. 11B, Academic Publishers

James, J. F., Mukai, T., Watanabe, T., Ishiguro, M., Nakamura, R. 1997, MNRAS, 288, 1022

Koch, D., Borucki, W., \& Cullers, K. 1996, JGR E, 101, 9297

Mayor, M., \& Queloz, D. 1995, Nature, 378, 355

Rouan, D., Baglin, A., Barge, P., Copet, E., Deleuil, M., Léger, A., Schneider, J., Toublance, \& Vuillemin, A. 1999, Phys. and Chemist. of the Earth 24, 567

Robichon, N., \& Arenou, F. 2000, A\&A, 355, 295

Rosenblatt, F. 1971, Icarus, 14, 71

Sartoretti, P., \& Schneider, J. 1998, A\&AS. 134, 553

Schneider, J. 1996, Ap\&SS, 241, 35

Wuchterl, G, Guillot, T., \& Lissauer, J. 1999, in "Protostars and Planets IV" (in press) 